\title{
Método de avaliação visual da qualidade da estrutura aplicado a Latossolo Vermelho Distroférrico sob diferentes sistemas de uso e manejo
}

\author{
Visual assessment soil quality structure methodology applied to Oxisol under different soil use \\ and management
}

\author{
Neyde Fabíola Balarezo Giarola' Cássio Antonio TormenaII Alvaro Pires da SilvaII \\ Bruce Ball ${ }^{\mathrm{IV}}$
}

-NOTA-

RESUMO

O aumento da demanda pela avaliação da qualidade da estrutura do solo para o adequado crescimento de plantas tem motivado pesquisadores a desenvolverem técnicas visuais de avaliação, a campo, simples e confiáveis para esse fim. No Brasil, um número reduzido de estudos foi realizado empregando métodos visuais de diagnóstico do estado estrutural de solos no campo. Esse trabalho testou a hipótese de que o método de Avaliação Visual da Qualidade da Estrutura do Solo desenvolvido por BALL et al. (2007) para solos de clima temperado pode ser aplicado na identificação de campo da qualidade estrutural de um Latossolo Vermelho Distroférrico sob diferentes sistemas de uso e manejo. Para isso, foram avaliadas amostras indeformadas coletadas de mata preservada $(M)$, sistema de integração lavoura-pecuária (ILP) e sistema plantio direto (SPD). A avaliação da estrutura apoiou-se na aparência, na resistência e nas características das unidades estruturais de blocos de solo e foi definida por cinco escores visuais de classificação de qualidade. O método empregado permitiu distinguir a qualidade do solo de diferentes sistemas de uso e manejo a partir da avaliação da estrutura da camada estudada.

Palavras-chave: qualidade física do solo, morfologia do solo, sistema plantio direto, sistema integração lavoura-pecuária.

\section{ABSTRACT}

The increasing demand for assessing soil structure for crop growth has motivated researchers to develop simple and reliable visual indicators to assess soil structure at the field. There are a few records in Brazil indicating the use of visual techniques for assessing soil physical quality. This paper tested the hypothesis that the Visual Soil Structure Quality Assessment methodology developed by BALL et al. (2007) is reliable for identifying the structural quality of tropical and subtropical soils under different soil management systems. Therefore, the overall objective of this paper was to visually identify the structural quality of an Oxisol under forest, croplivestock rotation and no-till system. The structure evaluation was based on appearance, strength and characteristics of structural units from soil blocks, and was defined by five visual scores from the classification quality. The method allows distinguishing soil quality of the different soil use and management systems from the evaluation of the layer sampled.

Key words: soil physical quality, soil morphology, no-till, croppasture rotation.

A qualidade estrutural do solo pode ser identificada por meio de avaliações visual e táctil, realizadas e concluídas diretamente no campo (BATEY \& MCKENZIE, 2006). Avaliações visuais do solo podem permitir o exame detalhado dos aspectos da estrutura e indicar as profundidades ou camadas mais relevantes para amostragens, para análises mais específicas (BATEY, 2000). Essas avaliações são, normalmente, expeditas e de baixo custo, o que não limita a diagnose de problemas físicos do solo.

As técnicas de avaliação visual da qualidade estrutural do solo evoluíram consideravelmente nos

\footnotetext{
'Departamento de Ciência do Solo e Engenharia Agrícola, Universidade Estadual de Ponta Grossa (UEPG), 84030-900, Ponta Grossa, PR, Brasil. E-mail: neydef@uepg.br. Autor para correspondência.

"Departamento de Agronomia, Universidade Estadual de Maringá (UEM), Maringá, PR, Brasil.

IIIDepartamento de Ciência do Solo, Escola Superior de Agricultura Luís de Queiroz (ESALQ), Universidade de São Paulo (USP), Piracicaba, SP, Brasil.

IvSAC Crop and Soil Systems Research Group, West Mains Road, Edinburgh EH9 3JG, UK
} 
últimos anos, especialmente na Europa e Oceania. Revisões, considerações e especificidades dessas metodologias podem ser obtidas nos trabalhos de BATEY (2000), BALL \& DOUGLAS (2003), BATEY \& MCKENZIE (2006) e BALL et al. (2007).

No Brasil, o método do Perfil Cultural (GAUTRONNEAU \& MANICHON, 1987) foi o único empregado no diagnóstico do estado estrutural de solos no campo (TAVARES FILHO et al., 1999; FREGONEZI et al., 2001). No entanto, o rigor e a complexidade da proposta metodológica inicial exigiram inúmeras adaptações e limitaram a adoção mais ampla do método.

O método denominado Avaliação Visual da Qualidade da Estrutura do Solo (BALL et al., 2007) tem permitido distinguir, com simplicidade e agilidade, camadas com diferenças estruturais em solos de textura franco-arenosa de regiões temperadas. A hipótese deste trabalho é que esse método de avaliação visual da estrutura é viável para identificar, a campo, a qualidade estrutural de solos argilosos de regiões tropicais sob diferentes condições de uso e manejo. O objetivo deste trabalho foi identificar, por meio do método visual de campo desenvolvido por BALL et al. (2007), a qualidade estrutural de um Latossolo Vermelho Distroférrico sob sistemas de uso e manejo característicos da região central do Paraná.

O estudo foi realizado na fazenda experimental da Cooperativa Agroindustrial de Campo Mourão(COAMO), localizada no Município de Campo Mourão, Paraná (PR), com coordenadas médias $24^{\circ} 02^{\prime} 38^{\prime \prime}$ S e $52^{\circ} 22^{\prime} 40^{\prime \prime} W$. Nessa região, as altitudes variam de 600-650m, e o tipo climático dominante é do tipo subtropical úmido mesotérmico (Cfa), com médias anuais de temperatura e precipitação de $20^{\circ} \mathrm{C}$ e $1.340 \mathrm{~mm}$, respectivamente (IAPAR, 2000). O solo utilizado no estudo foi classificado como Latossolo Vermelho Distroférrico e apresentava, na camada 0,00,15m, $850 \mathrm{~g} \mathrm{~kg}^{-1}$ de argila, $120 \mathrm{~g} \mathrm{~kg}^{-1}$ de silte e $30 \mathrm{~g} \mathrm{~kg}^{-1}$ de areia.

A primeira área amostrada se encontrava sob sistema integração lavoura-pecuária (ILP) desde 2002, conduzido em sistema plantio direto de aveia+azevém no inverno e soja ou milho no verão. A forragem de inverno (aveia+azevém) era mantida com 0,28m de altura durante o pastejo (ILP-28). A segunda área amostrada se encontrava sob sistema plantio direto (SPD) desde 1998, de modo que as culturas de inverno (aveia+azevém) eram utilizadas como plantas de cobertura e as de verão para a produção de grãos (milho ou soja). Como referência de qualidade estrutural do solo, utilizou-se uma área contígua sob floresta estacional semidecidual (M).
Com o auxilio de uma pá reta, foram abertas minitrincheiras com $0,30 \mathrm{~m}$ de largura x $0,40 \mathrm{~m}$ de comprimento $\mathrm{x} 0,30 \mathrm{~m}$ de profundidade, para a extração de amostras indeformadas (blocos) com 0,15m de largura $\mathrm{x} 0,25 \mathrm{~m}$ de profundidade $\mathrm{x} 0,10 \mathrm{~m}$ de espessura da camada superficial do solo de cada tratamento. Da ILP-28 e M, foram retirados 10 blocos e do SPD, quatro blocos, em razão do reduzido tamanho da área experimental deste. A avaliação da estrutura apoiou-se na aparência, na resistência e nas características das unidades estruturais de blocos de solo, sendo definida por cinco escores visuais (Ev) para a classificação da qualidade: $\mathrm{de} E v=1$ (melhor qualidade estrutural) a $\mathrm{Ev}=5$ (pior qualidade estrutural). A figura 1 exemplifica a atribuição de escores e o cálculo do escore final conforme a chave de classificação de BALL et al. (2007).

A operação de extração dos blocos de solo foi muito afetada pela umidade do solo e pela abertura de minitrincheiras. A necessidade da abertura da minitrincheira implicou esforço maior de trabalho, em tempo superior de amostragem por ponto (15 minutos, em média) e em perturbação considerável da área. Dessa forma, a metodologia apresentou restrições quando empregada em parcelas experimentais com dimensões reduzidas, em solos muito argilosos com teor de água abaixo da capacidade de campo e/ou com compactação do solo. A presença de pessoal com habilidade de trabalho braçal também foi essencial na equipe de trabalho. No trabalho apresentado por BALL et al. (2007), não houve menção a esse tipo de dificuldade para a retirada dos blocos de solo.

A proposta metodológica demandou habilidade para manipular e reconhecer aspectos da estrutura do solo. $\mathrm{O}$ aspecto mais importante foi o modo de individualizar as unidades estruturais, respeitando as linhas de definição dos agregados sem promover deformação ou formação de novos agregados. Essas particularidades indicaram que o observador necessita de conhecimento prévio sobre a morfologia da estrutura do solo maior que o indicado por BALL et al. (2007).

Em todos os pontos amostrados, no mínimo duas camadas de solo com diferenças estruturais foram facilmente identificadas, principalmente quando estavam presentes plantas com sistema radicular ativo. O efeito do sistema radicular de aveia+azevém foi denotado pela presença de uma camada superficial, composta por agregados de pequeno tamanho e ocupados por muitas raízes.

Ciência Rural, v.39, n.8, nov, 2009. 


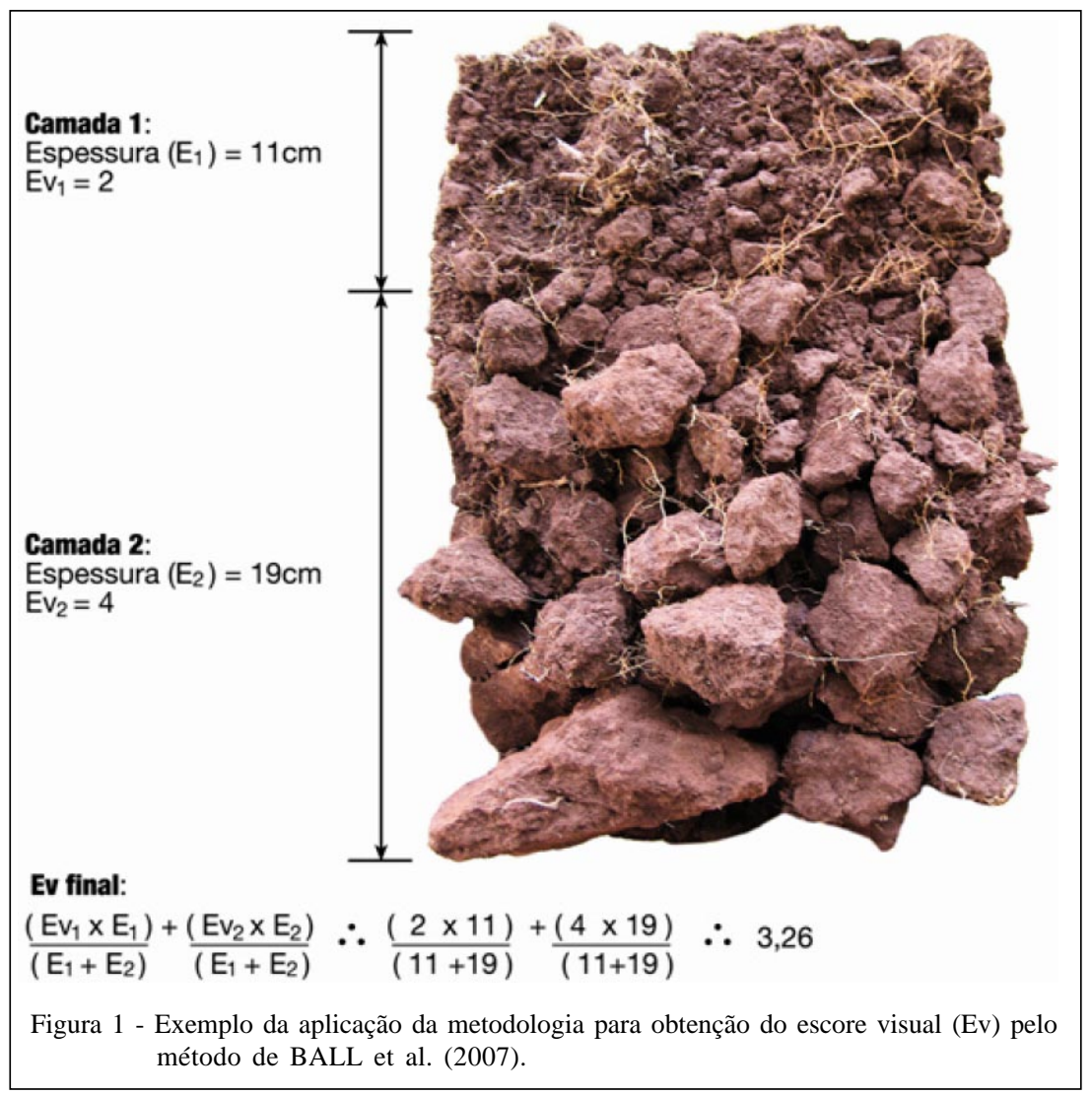

Fotografias com os aspectos estruturais do solo e os escores finais obtidos nos diferentes tratamentos são apresentadas nas figuras $2 \mathrm{a}$ e $2 \mathrm{~b}$, respectivamente.

Verificou-se mudança gradual e contínua da estrutura do solo, destacando-se a presença de camadas com diferentes espessuras, formas e tamanhos dos agregados, que resultaram num Ev menor na $\mathrm{M}$ $(E v=1,70 \pm 0,06)$, seguido do SPD $(E v=2,2 \pm 0,35)$ e ILP-

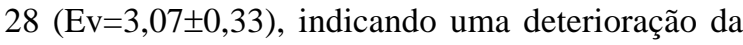
qualidade da estrutura do solo nessa sequência. Nos tratamentos ILP-28 e SPD, foram identificadas amostras com até três camadas e espessuras variáveis, apesar de que, na maioria delas, distinguiram-se claramente duas camadas. No solo sob mata, a variabilidade da estrutura das amostras foi muito pequena, indicando que a ausência de estresse mecânico manteve a homogeneidade estrutural e física do solo. As amostras coletadas nas áreas submetidas ao pastejo apresentaram agregados de maior tamanho e resistência à ruptura na maior parte destas. No solo sob SPD, cerca de 50\% da amostra apresentaram agregados de menor tamanho e com elevada friabilidade, situada na camada superficial. Em profundidade, apareceram agregados de maior tamanho, mais densos, os quais sugerem a presença de uma camada compactada decorrente, provavelmente, do preparo convencional utilizado antes da adoção do SPD ou, então, da consolidação do solo em função do tráfego na superfície.

De acordo com a proposta de BALL et al. (2007), os sistemas com escores entre 1 e 3 indicam condições aceitáveis de manejo e qualidade física do solo, o que se refletiu em todos os tratamentos avaliados. No sistema ILP-28 (Ev=3,07), seriam recomendadas avaliações periódicas para observar a evolução da qualidade física do solo com base no escore de avaliação visual da estrutura.

A hipótese de aplicabilidade da metodologia de avaliação visual da estrutura foi comprovada e indicou a exequibilidade desta na avaliação e no monitoramento da qualidade da estrutura do solo e na distinção de sistemas de manejo com diferentes qualidades estruturais, com ressalva para a dificuldade de coleta das amostras.

Ciência Rural, v.39, n.8, nov, 2009. 


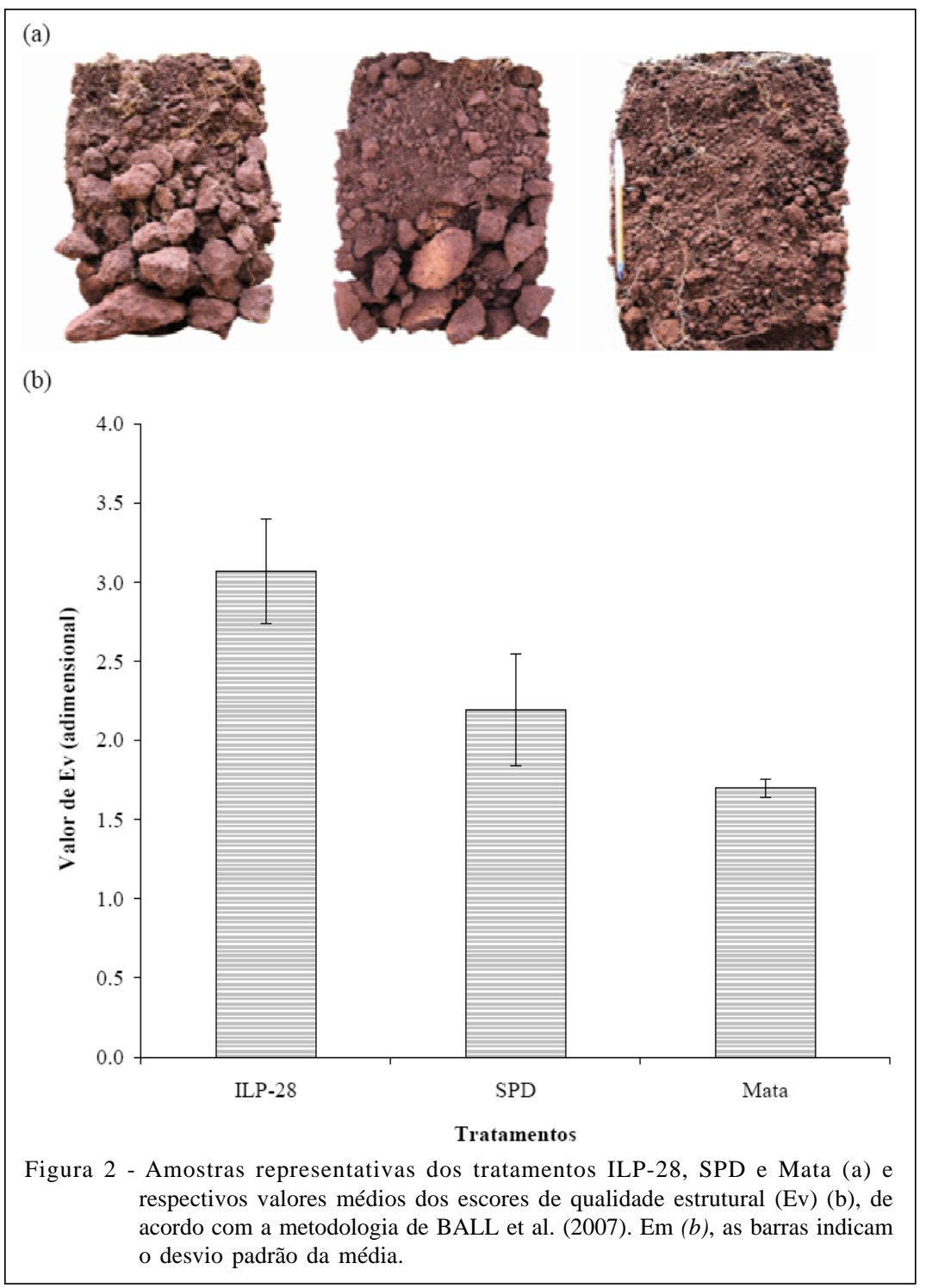

\section{REFERÊNCIAS}

BALL, B.C.; DOUGLAS, J.T. A simple procedure for assessing soil structural, rooting and surface conditions. Soil Use and Management, v.19, p.50-56, 2003. Disponível em: <http:// www.ingentaconnect.com/content/bpl/sum/2003/00000019/ 00000001/art00007>. Acesso em: 10 out. 2008. doi: 10.1111/ j.1475-2743.2003.tb00279.x

BALL. B.C. et al. Field assessment of soil structural quality - a development of the Peerlkamp test. Soil Use and Management, v.23, p.329-337, 2007. Disponível em: <http:/ /www.ingentaconnect.com/content/bpl/sum/2007/00000023/ 00000004/art00001?crawler=true>. Acesso em: 9 out. 2008. doi: $10.1111 / \mathrm{j} .1473-2743.2007 .00102 . x$

BATEY, T. Soil profile description and evaluation. In: SMITH, K.A.; MULLIN, C.E. Soil and environmental analysis: physical methods. New York: Marcel Dekker, 2000. p.595628.
BATEY, T.; McKENZIE, D.C. Soil compaction: identification directly in the field. Soil Use and Management, v.22, p.123131, 2006. Disponível em: <http://www.ingentaconnect.com/ content/bpl/sum/2006/00000022/00000002/art00002>. Acesso em: 11 out 2008. doi: 10.1111/j.1475-2743.2006.00017.x

FREGONEZI, G.A.F. et al. Modificações morfológicas e físicas de um Latossolo argiloso sob pastagens. Revista Brasileira de Ciência do Solo, v.25, p.1017-1027, 2001.

GAUTRONNEAU, Y.; MANICHON, H. Guide méthodique du profil cultural. Lyon: CEREF-GEARA, 1987. 71p.

INSTITUTO AGRONÔMICO DO PARANÁ - IAPAR. Cartas climáticas do Paraná: edição ano 2000, versão 1.0. Londrina, 2000. CD-ROM.

TAVARES FILHO, J. et al. Método do perfil cultural para avaliação do estado físico de solos em condições tropicais. Revista Brasileira de Ciência do Solo, v.23, p.393-399, 1999.

Ciência Rural, v.39, n.8, nov, 2009. 